\title{
DIE HERAUSBILDUNG DER GRAMMATIK BEIM FREMDSPRACHENLERNPROZESS (AUSGEWÄHLTE PROBLEMKREISE)
}

Die Herausbildung der Grammatik bei den Fremdsprachenlernenden ist nur ein Bestandteil des ganzheitlichen Lernprozesses; dieser Vorgang findet vor allem unter schulischen Unterrichtsbedingungen statt, wo sowohl Lehr- als auch Lerntätigkeiten ausgeübt und entwickelt werden.

Aus dieser Voraussetzung sind zwei wichtige Fragen abzuleiten. Die eine ist linguistischer Natur, die sich auf die Grammatik als Teil der Sprachwissenschaft bezieht, in dem die Bildung von Wortformen und demnach ihre möglichen Verbindungen zu Wortsyntagmen (Wortgruppen) und zu Sätzen erörtert und beschrieben werden. Die zweite hat hingegen einen fremdsprachendidaktischen Charakter, wo zum Untersuchungsgegenstand effektive Lehr- sowie optimale Erlernungs- und Aneignungsmethoden der grammatischen Mittel in schulischen Unterrichtssituationen behandelt und angewendet werden.

Denn gerade hierin liegt die Voraussetzung für die Beherrschung der Grammatik im Fremdsprachenunterricht. Das entspricht grosso modo der von Helbig (1981: 49 f.) konzipierten Trichotomie, wo die Grammatik A, B und C differenziert wird. In dieser Dreiteilung ist insbesondere die für unsere Erörterungen passende Konzeption bezüglich der Grammatik $\mathrm{C}$ hervorzuheben. Unter linguistisch-kodematischem Gesichtspunkt betrifft sie das interne Grammatik-Regelsystem, das sich im Gedächtnis des Lernenden während der phonetisch-phonematischen bzw. graphisch-graphematischen und semantisch-grammatischen Dekodierung der einlaufenden Signale in Form von grammatischen Strukturen, die sicherlich teilweise den fremdsprachlichen Speicher-Mechanismus konstituieren, stufenweise und allmählich herausbildet. Bekanntlich kann der fungierende Speicher-Mechanismus des Lernenden die empfangenen fremdsprachlichen Signale völlig dekodieren und im Weiteren neue Informationen kodieren, d.h. der Lernende ist einerseits als Hörer bzw. Leser im Stande, die fremdsprachlichen Signale in Informationen umzuwandeln und sie zu verstehen, andererseits verfügt er auf Grund der schon herausgebildeten Sprachstrukturmatri- 
zen über nötige fremdsprachliche Fähigkeiten und Fertigkeiten, Äußerungen oder Texte selbst zu produzieren.

Will man den Gedanken der trichotomischen Konzeption weiterführen, so muss auch die Rolle der Grammatik B für den und in dem Fremdsprachenunterricht hervorgehoben werden. Sie versteht sich - dem Helbigschen Entwurf nach (Helbig 1981: 50) - als Abbildung der Grammatik A in Form der wissenschaftlich-linguistischen Beschreibung des innewohnenden Regelsystems einer Sprache. Unter dem Begriff Grammatik A wird also das überindividuelle und abstrakte Regelsystem einer Sprache zusammengefasst, auf Grund dessen Wörter in ihren Formen verändert und zu Sätzen verbunden werden. Dementsprechend ist die Grammatik B sowohl für die Theorie als auch für die Praxis Ausgangspunkt und zugleich Grundlage für die Auswahl, Anordnung, Integrierung, Aufbereitung und weitere Entwicklung des fremdsprachlichen Unterrichtsmaterials in bestimmten syntagmatisch-syntaktischen Konstruktionen. Die wissenschaftlich-linguistischen und fremdsprachlich-didaktischen Abhängigkeitsverhältnisse zwischen den Grammatiken A, B und C lassen sich schematisch folgendermaßen darstellen:

Grammatik A

das innewohnende Regelsystem

der Sprache

und

das Untersuchungsobjekt

für die Grammatik B

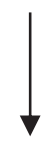

Grammatik B

die wissenschaftlich-linguistische Abbildung des innewohnenden Regelsystems der Sprache in Form dessen Beschreibung

und

die wissenschaftlich-linguistische

Grundlage für die Aufbereitung

des fremdsprachlichen Unterrichtsmaterials

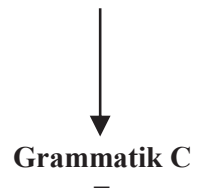

$=$

das herausgebildete (bzw. sich herausbildende)

interne Regelsystem im Gedächtnis des Lernenden/Sprachbenutzers

Abb. 1. Wissenschaftlich-linguistische Interrelationen zwischen den Grammatiken A, B, C 
Aus dem oben skizzierten Modell geht hervor, dass zwischen den Grammatiken $\mathrm{A}, \mathrm{B}$ und $\mathrm{C}$ glottodidaktische Interrelationen bestehen. Als Zwischenglied in den Relationen gilt die Grammatik B, welche relevante wissenschaftlich-linguistische wie auch glottodidaktisch-methodische Informationen den indirekten und den direkten Teilnehmern des Fremdsprachenunterrichts liefert, und zwar:

1. Dem/den Lehrbuch- bzw. Lehrwerkautor(en), von dem/den das in den Büchern/Werken der zu lehrenden und zu lernenden Fremdsprache Material bereitgestellt ist.

2. Dem Lehrenden, der das in jeder Unterrichtstunde zu lernende Material darbietet und dessen Übertragung durch entsprechende sprachliche sowie außersprachliche Steuerungs- und Regelungsmaßnahmen leitet und darüber hinaus den Zustand des dekodierten und gelernten Materials bei den Lernenden kontrolliert. Der Lehrende als direkter Fremdsprachenmittler muss unbedingt theoretisches Wissen über die Sprache wie auch über praktisches Wissen bezüglich der anzuwendenden Unterrichtsverfahren haben.

3. Letzten Endes auch die Lernenden selbst, weil die Aufbereitung und die Anordnung des zu lernenden Materials nicht nur während des Frontalunterrichts als optisch-visuelle Quelle und als Exposition (sowie Erklärung) der bestimmten semantisch-grammatischen Strukturen, sondern auch für ihre semiautonomen Tätigkeiten - beispielsweise - in der Gruppen- oder in der Partnerarbeit, aber vor allem für das außerunterrichtliche autonome Lernen sehr behilflich sein können und tatsächlich sind.

Um das Sprachpotential auf seine Funktionstüchtigkeit zu untersuchen, hat Darski (2003: 283 f.) eine dichotomische Konzeption hinsichtlich der analytischen und synthetischen Verarbeitung von sprachlichen Daten vorgelegt. Er vertritt die These, dass die im Gedächtnis durchgeführten sprachlichen Analysen und Synthesen auf Grund der sog. natürlichen Grammatik zu Stande kommen. Die wissenschaftlich-theoretischen Hypothesen über die möglichen Funktionen der natürlichen Grammatik sollte man - seines Erachtens - als linguistische Grammatik bezeichnen.

Glottodidaktische Konzeptionen hinsichtlich der Grammatik-Herausbildung bei Fremdsprachenlernenden sollen auch im Rahmen der Beherrschung von grammatischer Kompetenz reflektiert und diskutiert werden. Von dem Vorgang der Grammatik-Herausbildung und deren Ergebnissen sind die Fähigkeiten und die Fertigkeiten im Bereich der grammatischen Kompetenz abhängig, die sich vor allem in der mündlichen und in der schriftlichen Produktion erkennbar machen. Als wesentliche Eigenschaften der fremdsprachlichen Kompetenz zeigen sich - angesichts der von Hellmich (1981: 80) formulierte Theorie - ihre einzelnen Herausbildungs- und Entfaltungsstufen und das Niveau der fremdsprachlichen Tätigkeiten, die das fremdsprachenkommunikative Können widerspiegeln. Dem fremdsprachenkommunikativen Können liegen jedoch die sprachlichen Tätigkeiten zu Grunde, und das sprachliche Können tritt in Fähigkeiten und Fertigkeiten wie auch in manchen Fällen 
in Kenntnissen zu Tage. Gemeint sind hier einerseits mögliche Vernetzungen zwischen sprachlichen Einheiten, die sowohl aus lexikalischen als auch aus grammatischen Strukturen bestehen, und andererseits die Vorkommens-Wahrscheinlichkeit der sprachlichen Einheiten von möglichen Kombinierbarkeiten im Bereich der syntagmatisch-syntaktischen Konstruktionen. $\mathrm{Zu}$ solchen Leistungen trägt die Konstruktionsgrammatik bei, die zu den deklarativen Grammatikansätzen gerechnet wird und semantisch-grammatisch realisierbare Zusammenhänge als Repertoire unterschiedlicher Strukturformen darstellt und solche im Weiteren erzeugen kann (siehe dazu Dürscheid 2010: 58; Herbst 2010: 203f.). Auf die syntaktischen Relationen bezieht sich gleichermaßen das synaptische Netzwerk, das die einzelnen Syntaxelemente als System von deren Verbindungen betrachtet und auch als Steuerungswerk angesehen wird; dieses Leistungsvermögen wird dementsprechend unter dem Begriff der sog. Netzwerkgrammatik subsumiert (vgl. Batori 1981: 69).

Daneben gibt es selbstverständlich auch eine andere - konstruktivistische - Auffassung der Sprache, die nicht nur als deklaratives Wissen über das grammatische Regelsystem und den Wortschatz, sondern auch als Regelsystem des kommunikativsozialen Verhaltens begriffen wird, das für die bestimmte Sprachgemeinschaft gilt. Somit wird hier die konstruktivistische Auffassung im doppelten Sinne vertreten, nämlich beim Erwerb der Sprache lernt man zugleich die Konventionen der sozialen Verhaltensnormen, die von den Mitgliedern der Sprachgemeinschaft vereinbart worden sind (Steinkrauss 2010: 298). Wenn die (Fremd-)Sprache parallel im kommunikations-situativ-sozialen Kontexten erlernt/erworben wird, so findet die Kodierung der Sprachstrukturen auf Grund von automatisiertem Können statt, d.h. von unmittelbarer Anpassung der grammatischen Mittel an die lexikalischen Einheiten.

Im Fremdsprachenunterricht machen sich - und wahrscheinlicht nicht so selten - die Lernenden Gedanken über die effektiven Aneignungsmöglichkeiten der zu lernenden und über die erfolgreiche sowie richtige Anwendung der schon gelernten Strukturen, d.h. sowohl die Lehrenden als auch die Lernenden brauchen eine klarere Auslegung der bestimmten grammatischen Mittel für schulische Unterrichtszwecke und für den kommunikationssituativen Sprachgebrauch. Eine gründlichere Erörterung der bereits angeschnittenen Probleme bringt zwei weitere Fragen linguistischglottodidaktischer Natur mit sich, die man nicht unterschätzen darf. Als Voraussetzung ist sicherlich die wesentliche Unterscheidung notwendig, ob nämlich im Fremdsprachenunterricht nur das grammatische Können durch die Lernenden herausgebildet werden soll oder ob sie auch das grammatische Wissen für die Erlernung und Anwendung des sprachlichen Materials brauchen. Wenn beide Verfahren angewendet werden, so taucht zugleich eine weitere Frage auf, und zwar: In welchen didaktisch-methodischen Verhältnissen stehen die beiden Verfahren zueinander und welche Aufgaben sind zu verwirklichen, um die gesetzten Ziele zu erreichen? Das grammatische Können ist jedoch als untrennbare Komponente des erlernten/erworbenen Vermögens im Bereich der sprachlichen Fähigkeiten und Fertigkei- 
ten zu betrachten, um benötige und korrekte mündliche bzw. schriftliche Sprachinformationen produktiv zu erbringen oder die dekodierten Sprachinformationen vom Standpunkt ihrer grammatischen Richtigkeit aus zu bewerten. Das grammatische Können erlernt/erwirbt man im Prozess des mehrmaligen Wiederholens/Aktivierens der grammatischen Strukturen, auf Grund dessen sie in Form von rezeptivproduktiven Strukturmatrizen so ins Langzeitgedächtnis (in den sprachlichen Speicher-Mechanismus) eingeprägt werden, dass die grammatischen Strukturen sowohl während der Dekodierung als auch während der Kodierung von Sprachinformationen unbewusst und automatisch angewendet werden. Die Generierung der Sprachinformationen im Speicher-Mechanismus wird unter zwei Aspekten gesehen: Erstens findet der Suchprozess nach den für die Kommunikationsabsicht benötigen Wörtern statt; er kennzeichnet sich durch eine bewusste Handlung. Zweitens vollzieht sich die Anordnung der einzelnen aufgefundenen Wörter und im Weiteren deren richtige grammatische Verbindungen in Satzkonstruktionen vollständig automatisch (Leiss 2010: 291; Szczodrowski 2011: 97).

Das grammatische Wissen versteht sich einerseits als deklaratives Wissen über Gesetzmäßigkeiten, welche sich auf innere Ordnungsprinzipien der Strukturen einer Sprache beziehen, andererseits auch als Wissen über aus bestimmten Gesetzmäßigkeiten abgeleitete und festgelegte Regeln, die im Bereich der Morphologie und Syntax gelten. Es handelt sich also in diesem Fall um explizites Wissen, das in Form von verbindlichen Normen und Vorschriften auf metasprachliche Weise graphisch dargestellt und im Fremdsprachenunterricht seitens der Lernenden bewusst reflektiert wird (Näheres hierzu u.a. bei Wollf 1990: 614; Habermann 2010: 21; Thurmair 2010: 357).

Mit der Komplexität der grammatischen Phänomene beschäftigt sich die wissenschaftlich-deskriptive Grammatik, von der die sogenannte Schulgrammatik abgeleitet wurde und zu deren Grund die didaktisch-präskriptive Zielsetzung liegt. Die Schulgrammatik beschäftigt sich besonders mit zwei Unterrichtsproblemen, nämlich zunächst mit der Gestaltung und der Anordnung der grammatischen Mittel im Bereich der Unterrichtsmaterialien in den Lehr-Lern-Büchern/Werken, dann aber auch mit den Vermittlungs- und Erlernungsverfahren dieser Mittel in schulischen Unterrichtssituationen. In den zwei Aufgaben der Schulgrammatik ist sowohl die linguistische als auch die glottodidaktische Relevanz zu berücksichtigen (Ganslmayer 2010: 31).

Für die wissenschaftlich-theoretischen und die glottodidaktisch-praktischen Aufbereitungs- und Anordnungsprinzipien der Unterrichtsmaterialien gelten lexikalische und grammatische Strukturen als sprachliche Einheiten, die im Unterricht vermittelt und gelernt werden. In jedem theoretisch-praktischen Konzept wird von den notwendigen Voraussetzungen ausgegangen, dass sowohl in der Auswahl als auch in der Gestaltung der Unterrichtsmaterialien die linguistisch-didaktischen Grundsätze beachtet werden müssen, nach denen man die Materialien von den Wör- 
tern und deren Verknüpfungen zu Syntagmen und Sätzen für festgelegte glottodidaktische Zwecke aufbaut. Wie sich die Wort-Junktionen (Wort-Verknüpfungen) vollziehen, kann hier kurz dargestellt werden: Die für die Generierung der Sprachinformationen im Speicher-Mechanismus ausgewählten einzelnen Wörter bestimmen schon teilweise grammatische Beziehungen, auf Grund deren sie zu größeren Einheiten (Syntagmen) und diese zu Satzkonstruktionen verbunden werden (Fritz 2010: 239). Die bereits genannten Sprachstrukturen in Form von Wort- und Satzeinheiten sind für das Unterrichten selbstverständlich graphisch in entsprechenden syntagmatisch-syntaktischen Kontexten dargestellt und werden von den Unterrichtsteilnehmenden phonetisch-phonologisch - in manchen Fällen auch graphisch-graphematisch realisiert, wodurch man es in unterschiedlichem Ausmaß mit vielen (allen!) für die Erlernung nötigen und möglichen Sprachstrukturformen zu tun hat.

Jede Gestaltung der Unterrichtsmaterialien berücksichtigt auch ihre grammatischen Komponenten, die nicht nur für deren Autor(en) von besonderer Bedeutung sind, sondern auch für die Lehrenden. Beide als indirekte und direkte Unterrichtsgestalter brauchen hinreichendes Grammatikwissen über die sprachlichen Regeln und ihre Ausnahmen, über ihren Schwierigkeitsgrad wie auch über ihre Häufigkeit und ihre Reichweite im situativ angemessenen Sprachgebrauch. Gerade diese Probleme erfordern eine durchdachte Progression der Grammatik sowie auch eine anschauliche (bildhafte) Darstellung und Erklärung, um die Aufmerksamkeit der Lernenden während der Darbietung des Sprachmaterials auf bestimmte grammatische Phänomene zu konzentrieren. Schliesslich darf nicht unerwähnt bleiben, dass kontrastiv angelegte grammatische Konstruktionen den Fremdsprachenlernenden als wirksame Gedächtnisstütze dienen und somit den Lernprozess optimieren können (vgl. mehr dazu Thurmair 2010: 359 ff.). Konfrontativ-kontrastive Sprachkonstruktionen können für den Erlernungsprozess als zweckmäßig konzipierte Grammatikstrukturen Kenntnisse liefern, welche Strukturen richtig und möglich oder welche fehlerhaft und unmöglich sind. Will man ausgewählte grammatische Strukturen des Sprachmaterials in konfrontativ-kontrastiver Weise anordnen, so sind wissenschaftliche Ergebnisse der konfrontativ-kontrastiven Studien vorzulegen, die es ermöglichen, die Lehr-Lern-Materialien auf der bilateralen externen oppositionellen Basis aufzubauen (vgl. ausführlicher bei Zabrocki 1972: 4f.).

In den fremdsprachenunterrichtlichen Anwendungsbereichen, und zwar bei der Konzipierung und Gestaltung des Sprachstoffes in den Lehr-Lern-Büchern/Werken sowie während der Lehr-Lern-Prozesse haben - so Klein 2010: 102ff. - die grammatischen Regeln eine besondere Relevanz, deren Wesen gerade die Präskription berücksichtigen muss. Klein bezieht sich im Weiteren auf die für den Fremdsprachenunterricht implizite und explizite Wichtigkeit sowohl der deskriptiven als auch der präskriptiven Grammatik. Aus theoretischen Erörterungen sind seines Erachtens folgende Feststellungen abzuleiten: Erstens wird in der wissenschaftlichen Forschung über Sprache (auch Grammatik) deskriptiv reflektiert, zweitens ist Deskrip- 
tivität ohne Präskriptivität nicht zu haben, und drittens sind deskriptive Grammatikregeln schon mit Präskriptivitätsgehalten aufgeladen. Diese Ausführungen weisen einerseits auf ihre theoretische Relevanz bezüglich der Problemstellungen für den effektiven Fremdsprachenunterricht hin und andererseits gehen sie auf praktische Lösungsmöglichkeiten im Bereich des Lehrens und des Lernens zurück.

Bei der Integrierung und der Aufbereitung des Fremdsprachenmaterials für unterrichtliche Zwecke wird auch darüber reflektiert, wie und inwieweit Grammatik in den Unterrichtsmaterialien systematisch und gradual erstellt sein soll und auf Grund welcher wissenschaftlichen Theorie die syntaktischen Konstruktionen darzustellen sind (Haueis 1981: 9).

In jedem Sprachlernvorgang handelt es sich nicht nur um eine Ansammlung und Aufspeicherung von Wörtern, sondern auch um die Herausbildung eines solchen Mechanismus, der fähig ist, sprachliche Signale in Form von Äußerungen bzw. Texten zu empfangen und sie zu verstehen sowie sprachliche Informationen in Form von Äußerungen bzw. Texten selbst zu produzieren. Die rezeptiv-produktiven Fähigkeiten und die Fertigkeiten als Eigenschaften des sprachlichen SpeicherMechanismus kommen in der zwischenmenschlichen Kommunikation zum Ausdruck, wo man es vor allem mit Informationen in Form von Sätzen zu tun hat. Die Satzformen, welche aus sprachtheoretischer Sicht als rekonstruierte Abbildungen der sprachlichen Wirklichkeit und unter anwendungsbezogenem Aspekt als Muster für die Gestaltung des Unterrichtsstoffes gelten, sind geeignete sprachliche Einheiten, auf Grund deren man Einsichten gewinnt, wie die einzelnen Wörter in verschiedenartigen Wechselbeziehungen auf geordnete Weise miteinander und nebeneinander verkettet werden. Die vielfältig möglichen Wechselbeziehungen und Vernetzungen von Wörtern, in denen sich die grundlegenden Strukturen einer Sprache widerspiegeln, bilden eine feste Basis für deren optimale und effektive Erlernung und Aneignung (vgl. dazu u.a. Steinmüller 1977: 38; Weisgerber 1985: 119; Krais 1990: 15; Dürscheid 2010: 58).

Werden Sprachinformationen als größere Äußerungs- bzw. Textzusammenhänge, die aus bestimmten sich überlagernden Strukturen bestehen, betrachtet, dann erfüllt die Grammatik in solchen Wort- und Satzverbindungen selbstverständlich ihre kommunikationskonstituierende und - koordinierende und zugleich eine entscheidende kommunikationssteuernde Funktion (mehr dazu Gürtler 1981: 29 u. 37).

Während der ganzheitlichen Dekodierung der im Gedächtnis empfangenen Sprachsignale werden alle ihre Komponenten miteinbezogen und hinsichtlich der Informationsstrukturen analytisch-synthetisch verarbeitet, d.h. die Informationsstrukturen werden im Speicher-Mechanismus so semantisch und grammatisch strukturiert, dass sich in ihm das sog. syntaktische Verstehen vollzieht (Strohner 1990: 30).

Abschließend seien hier nur noch einige Anmerkungen zum Verhältnis von Linguistischer Grammatik und Didaktischer Grammatik angeführt. Die Linguistische Grammatik beschäftigt sich - so wie es eingangs kurz formuliert wurde - mit der 
Deskription der Bildung verschiedener Wortformen und deren Funktionen in Sätzen, und die Didaktische Grammatik dagegen geht von der unterrichtspraktischen Relevanz aus und bereitet dem eigensprachlichen und dem fremdsprachlichen Unterricht besonders solche Grammatikstrukturen vor, die den festgelegten Lernbedingungen entsprechen und durch die Lernenden angeeignet werden sollen (Jung 1975: 7, 33). In der sachlichen Diskussion über das Wesen der Aufbereitung, Entwicklung und Integrierung des Unterrichtsmaterials wird immer folgende Frage vorgebracht: Aus welcher grammatischen Perspektive geht man an die Unterrichtsmaterial-Gestaltung heran? Die von Zabrocki (1969: 68) aufgestellte Theorie beruht auf der Annahme, dass man sich nicht ausschließlich auf eine der vielen ausgearbeiteten GrammatikModelle/Arten stützen kann. Die Lösung der recht komplizierten Probleme sollte man - seiner Meinung nach - auf der multigrammatikalischen Basis suchen und finden, wobei das Grundziel bei der Erlernung einer Fremdsprache unbedingt berücksichtigt werden muss, nach dem die zu lernende Grammatik so in entsprechenden Texten, Übungen sowie in paradigmatischen und syntaktischen Mustern eingefügt wird, dass man sie auf eine methodische Weise beim Kodieren und Dekodieren von konkreten Sätzen erlernen und beherrschen kann.

Es ist jedoch erforderlich festzuhalten, dass die Grammatik in den sich vollziehenden fremdsprachlichen Unterrichtsabläufen nicht unberücksichtigt bleibt. Wenn sie ebenso im engeren Sinne als Erklärung von Formen und Funktionen der Sprache $\mathrm{zu}$ verstehen ist, wird sie auch zur Herausbildung und zur Entwicklung des für das Kommunizieren nötigen grammatischen Könnens höchstwahrscheinlich beitragen.

\section{Literatur}

Batori, I.S. (1981): Die Grammatik aus der Sicht kognitiver Prozesse. Tübingen: Gunter Narr Verlag. Darski, J. (2003): Vielfalt von Sprachen - Vielfalt von Theorien? In: Deutsch-polnische und gesamteuropäische Integration in Forschung, Lehre und Öffentlichkeitsarbeit der (polnischen) Germanistik. Materialien der Jahrestagung des Verbandes Polnischer Germanisten 02.-04. Mai 2003, Szczecin. Hrsg. von F. Grucza. Warszawa: Wydawnictwo Euro-Edukacja, S. 283-293.

Dürscheid, Ch. (2010): Lateinische Schulgrammatik oder andere Modelle? Welche Grammatik eignet sich am besten zur Beschreibung des Deutschen? In: Grammatik wozu? Vom Nutzen des Grammatikwissens in Alltag und Schule. Thema Deutsch. Band 11. Hrsg. von M. Habermann. MannheimZürich: Dudenverlag, S. 47-65.

Fritz, T.A. (2010): Grammatik und Text. In: Grammatik wozu? Vom Nutzen des Grammatikwissens in Alltag und Schule. Thema Deutsch. Band 11. Hrsg. von M. Habermann. Mannheim-Zürich: Dudenverlag, S. 238-248.

Ganslmayer, Ch. (2010): Tradition und Entwicklung einer deutschen Grammatik. In: Grammatik wozu? Vom Nutzen des Grammatikwissens in Alltag und Schule. Thema Deutsch. Band 11. Hrsg. von M. Habermann. Mannheim-Zürich: Dudenverlag, S. 31-46.

Gürtler, I. (1981): Kontrastive Grammatik, kommunikativ. Tübingen: Gunter Narr Verlag. 
Habermann, M. (2010): Was ist eigentlich „Grammatik“? - Eine Einführung. In: Grammatik wozu? Vom Nutzen des Grammatikwissens in Alltag und Schule. Thema Deutsch. Band 11. Hrsg. von M. Habermann. Mannheim-Zürich: Dudenverlag, S. 9-14.

Haueis, E. (1981): Grammatik entdecken. Paderborn-München-Wien-Zürich: Ferdinand Schöningh. Helbig, G. (1983): Sprachwissenschaft - Konfrontation - Fremdsprachenunterricht. Zur Theorie und Praxis des Deutschunterrichts für Ausländer. Leipzig: VEB Verlag Enzyklopädie.

Hellmich, H. (1981): Stufen der fremdsprachlichen Kompetenz und das Niveau der (fremd)sprachliche Tätigkeit. In: Deutsch als Fremdsprache Nr. 2. Leipzig: Herausgeber Herder-Institut, S. 80-87

Herbst, T. (2010): Grammatik und Lexis. In: Grammatik wozu? Vom Nutzen des Grammatikwissens in Alltag und Schule. Thema Deutsch. Band 11. Hrsg. von M. Habermann. Mannheim-Zürich: Dudenverlag, S. 193-205.

Jung, L. (1975): Linguistische Grammatik und Didaktische Grammatik. Frankfurt am Main-BerlinMünchen: Moritz Diesterweg Verlag.

Klein, W.P. (2010): Grammatik zwischen Deskription und Präskription. In: Grammatik wozu? Vom Nutzen des Grammatikwissens in Alltag und Schule. Thema Deutsch. Band 11. Hrsg. von M. Habermann. Mannheim-Zürich: Dudenverlag, S. 97-111.

Krais, A. (1990): Fremdsprachen richtig lernen. Ehningen bei Böblingen: Expert Verlag.

Leiss, E. (2010): Das Zusammenspiel von mentaler Grammatik und mentalem Lexikon. In: Grammatik wozu? Vom Nutzen des Grammatikwissens in Alltag und Schule. Thema Deutsch. Band 11. Hrsg. von M. Habermann. Mannheim-Zürich: Dudenverlag, S. 286-294.

Steinkrauss, R. (2010): Die Entwicklung der Grammatik beim Erstspracherwerb. In: Grammatik wozu? Vom Nutzen des Grammatikwissens in Alltag und Schule. Thema Deutsch. Band 11. Hrsg. von M. Habermann. Mannheim-Zürich: Dudenverlag, S. 295-313

Steinmüller, U. (1977): Kommunikationstheorie. Eine Einführung für Literatur- und Sprachwissenschaften. Stuttgart-Berlin-Köln-Mainz: W. Kohlhammer Verlag.

Strohner, H. (1990): Textverstehen. Kognitive und kommunikative Grundlagen der Sprachverarbeitung. Westdeutscher Verlag, Opladen.

Szczodrowski, M. (2011): Von der grammatischen Dekodierung zur grammatischen Kompetenz. In: Grammatik im Text und im Diskurs. M. Wierzbicka, Z. Wawrzyniak (Hrsg.). Danziger Beiträge zur Germanistik, Band 34. Hrsg. von A. Kątny, P. Lang. Frankfurt am Main-Berlin-Bern-BruxellesNew York-Oxford-Wien: Internationaler Verlag der Wissenschaften, S. 91-99.

Thurmair, M. (2010): Grammatikwissen und Fremdsprachenerwerb: wer, was und wozu? In: Grammatik wozu? Vom Nutzen des Grammatikwissens in Alltag und Schule. Thema Deutsch. Band 11. Hrsg. von M. Habermann. Mannheim-Zürich: Dudenverlag, S. 357-370.

Weisgerber B. (1985): Vom Sinn und Unsinn der Grammatik. Bonn-Bad Godesberg: Dürrsche Buchhandlung Verlag.

Wollf, D. (1990): Zur Bedeutung des prozeduralen Wissens bei Verstehens- und Lernprozessen im schulischen Fremdsprachenunterricht. In: Die Neueren Sprachen 89, S. 610-625.

Zabrocki, L. (1969): Die Entwicklung und Integrierung des Unterrichtsmaterials. In: Probleme des Deutschen als Fremdsprache. Forschungsberichte und Diskussionen. Bericht von der 1. Internationalen Deutschlehrertagung 1967 in München. München: Max Hueber Verlag, S. 56-79.

Zabrocki, L. (1972): Zur Theorie der Aufbereitung des Sprachmaterials im Fremdsprachenunterricht. In: Glottodidactica, vol. VI, S. 3-39. 